\title{
QUASI-FROBENIUS QUOTIENT RINGS OF GROUP RINGS
}

\author{
ANDREAS HORN
}

(Received 19 March 1974)

\section{Introduction}

The purpose of this paper is an extension of a theorem of Hughes (1973). He showed:

Let $R$ be a ring which has a right artinian right quotient ring and let $G$ be a group which has a (transfinite) ascending normal series with each factor either finite or cyclic, but only a finite number of finite factors. Then the group ring $R G$ has a right artinian right quotient ring.

In the situation of Hughes' theorem we prove as the main result (Theorem 2.6) of this article that

$R G$ is an order in a quasi-Frobenius ring ( $Q F$ ring) if $R$ is an order in a $Q F$ ring.

This is also a generalization of our result (Horn $(1973 ; 3.9)$ ) that for a polycyclic-by - finite group $G$ the group ring $R G$ is an order in a $Q F$ ring if $R$ is an order in a $Q F$ ring. The proof of Theorem 2.6 is based on the results and methods developed in Horn (1973). In particular we obtain a different proof of Hughes' theorem.

In the following all considered rings have an identity. $J(R)$ denotes the Jacobson radical of the ring $R$. Let $\rho$ be an automorphism of $R$. Then $R[x, \rho]$ is as usual the skew polynomial ring over $R$.

Let $G$ be a group with normal subgroup $N$ such that $G / N$ is infinite cyclic. If $G / N$ is generated by $x N$ for some $x \in G$ let $\rho_{x}: R N \rightarrow R N$ be the automorphism of $R N$ defined by $\rho_{x}(a):=x^{-1} a x$ for all $a \in R N$. Then the quotient ring of $R G$ is up to isomorphism the quotient ring of $R N\left[x, \rho_{x}\right]$ when it 
exists (see Horn (1973; page 39)), furthermore it is the quotient ring of $Q\left[x, \rho_{\mathrm{x}}\right]$ where $Q$ is the quotient ring of $R N$.

The direct limit of a directed system $\left\{R_{\alpha} \mid \alpha<\gamma\right\}$ of rings $\boldsymbol{R}_{\alpha}$ ( $\gamma$ a limit ordinal) is written $R_{\gamma}=\lim _{\alpha<\gamma}\left(R_{\alpha}\right)$. Tacitly we assume that in a directed system for any $\alpha_{1} \leqq \alpha_{2}<\gamma$ always $R_{\alpha_{1}}$ is contained in $R_{\alpha_{2}}$, both rings have the same identity, and the ring homomorphism $R_{\alpha_{1}} \rightarrow R_{\alpha_{2}}$ given by the directed system is the inclusion. Then we identify the direct $\operatorname{limit} \lim _{\alpha<\gamma}\left(R_{\alpha}\right)$ with the union $\bigcup_{\alpha<\gamma} R_{\alpha}$.

For the definition of an ascending normal series of a group we refer to Kurosh (1956). All unexplained notation and terminology is as in Horn (1973).

I would like to thank Professor G. Michler for his suggestions. I am also indebted to the referee for valuable improvements.

\section{Proof of the main result}

The proof of Theorem 2.6 depends heavily on the following results contained in the paper Horn (1973). Theorem 2.1 generalizes a useful theorem of Shock (1972; Proposition 2.4).

TheоReм 2.1. Let $R$ be a ring with an automorphism $\rho$. The right ideal $A$ of $R$ is uniform in $R$ if and only if $A R[x, \rho]$ is a uniform right ideal of $R[x, \rho]$.

Proof. See Horn (1973; Satz 1.7).

Lemma 2.2. Let $R$ be a ring and let $G$ be a group with a normal subgroup $N$ such that $G / N$ is finite or cyclic. Then

(a) If $R N$ is a right order in a right artinian ring then $R G$ is also a right order in a right artinian ring.

(b) If $R N$ is an order in a $Q F$ ring then $R G$ is also an order in a $Q F$ ring.

Proof. See Horn (1973; Lemmas 3.2 and 3.8).

Before we now proceed to prove the main result we state two auxiliary lemmas.

Lemma 2.3. Let $\gamma$ be a limit ordinal and let $\left\{R_{\alpha} \mid \alpha<\gamma\right\}$ be a directed system of rings $R_{\alpha}$. Then

(a) If the regular elements of $R_{\alpha}$ are regular in $R_{\alpha+1}$ and the right quotient ring $Q_{\alpha}$ of $R_{\alpha}$ exists for every $\alpha<\gamma$, then $R_{\gamma}=\lim _{\alpha<\gamma}\left(R_{\alpha}\right)$ is a right order in $Q_{\gamma}=\lim _{\alpha<\gamma}\left(Q_{\alpha}\right)$.

(b) If $U$ is a uniform right ideal of $R_{0}$ such that $U R_{\alpha}$ is a uniform right ideal of $R_{\alpha}$ for all $\alpha<\gamma$ then $U R_{\gamma}$ is a uniform right ideal of $R_{\gamma}$.

Proof. (a) is straightforward.

(b). Suppose $U R_{\gamma}$ is not a uniform right ideal of $R_{\gamma}$. Then there are 
elements $0 \neq a, b \in U R_{\gamma}$ such that $a R_{\gamma} \cap b R_{\gamma}=0$. Hence for some ordinal $\beta<\gamma$ we have $a, b \in U R_{\beta}$. Thus $0 \neq a R_{\beta}, b R_{\beta} \leqq U R_{\beta}$ and $a R_{\beta} \cap b R_{\beta}=0$. This contradicts the uniformity of $U R_{\beta}$ as a right ideal of $R_{\beta}$.

Lemma 2.4. Let $R$ be a ring and let $G$ be a group with a (transfinite) ascending normal series $\left\{G_{\alpha} \mid \alpha \leqq \gamma\right\}$ from the subgroup $G_{0}$ to $G=G_{\gamma}$ ( $\gamma$ an ordinal) such that for every $\alpha<\gamma$ the factor group $G_{\alpha+1} / G_{\alpha}$ is infinite cyclic and $R G_{\alpha}$ is a right order in a right artinian ring $Q_{\alpha}$. Then

(a) $R G_{\gamma}$ has a right quotient ring $Q_{\gamma}$ and $Q_{\alpha}=\lim _{\beta<\alpha}\left(Q_{\beta}\right)$ for every limit ordinal $\alpha \leqq \gamma$.

(b) If $U$ is a uniform right ideal of $Q_{0}$ then $U Q_{\alpha}$ is a uniform right ideal of $Q_{\alpha}$ for all $\alpha \leqq \gamma$.

(c) $J_{\alpha}=J\left(Q_{\alpha}\right)=J\left(Q_{0}\right) Q_{\alpha}$ for all $\alpha \leqq \gamma$ and $J_{\alpha}=\lim _{\beta<\alpha}\left(J_{\beta}\right)$ for every limit ordinal $\alpha \leqq \gamma$.

(d) $J\left(Q_{0}\right)^{k}=J_{0}^{k}=0$ implies $J_{\alpha}^{k}=0$ for all $\alpha \leqq \gamma$.

(e) $\bar{Q}_{\alpha}=Q_{\alpha} / J_{\alpha}$ is a semisimple artinian ring for every $\alpha \leqq \gamma$.

Proof. We prove the statements by transfinite induction on $\alpha$. (a) follows immediately from the preceding Lemma 2.3 (a) and Lemma 2.2, (b) is a direct consequence of Theorem 2.1 and Lemma 2.3 (b), and (d) follows from (c).

For the proof of (c) and (e) first suppose that $\lambda$ is not a limit ordinal and that for $\alpha<\lambda$ (c) and (e) are valid. Then the assertions hold also for $\lambda$ : (c) follows from Korollar 2.10 of Horn (1973) and Theorem 1.9 of Small (1966) while Lemma 2.2 (a) yields (e).

Now we assume that $\lambda$ is a limit ordinal. By induction hypothesis we have $J_{\alpha+1}=J_{\alpha} Q_{\alpha+1}$ for all $\alpha<\lambda$. Since $J_{\alpha+1}$ is nilpotent it follows that $J_{\alpha+1} \cap Q_{\alpha}=J_{\alpha}$ and $K \cap Q_{\alpha}=J_{\alpha}$ with $K=\bigcup_{\alpha<\lambda} J_{\alpha} \subseteq J_{\lambda}$. Therefore $Q_{\lambda} / K$ is a direct limit of the von Neumann regular rings $K+Q_{\alpha} / K \cong Q_{\alpha} / J_{\alpha}$ for $\alpha<\lambda$, whence $Q_{\lambda} / K$ has the same property and therefore has zero Jacobson radical. Hence $K=J_{\lambda}$, which implies (c). Moreover from Lemma 2.3 we obtain that for a decomposition $1=e_{1}+\cdots+e_{n}$ with primitive orthogonal idempotents $e_{1}, \cdots, e_{n}$ in $Q_{0}$ the right ideal $e_{i} \bar{Q}_{\lambda}$ of $\bar{Q}_{\lambda}=Q_{\lambda} / J_{\lambda}$ is uniform in $\bar{Q}_{\lambda}$ for every $i=1, \cdots, n$. Therefore the ring $\bar{Q}_{\lambda}$ has finite right Goldie dimension. Thus we conclude that $\bar{Q}_{\lambda}$ is a semisimple artinian ring.

THEOREM 2.5 (Hughes (1973)). Let $R$ be a ring which is a right order in a right artinian ring and let $G$ be a group which has a (transfinite) ascending normal series with each factor either finite or cyclic, but only a finite number of finite factors. Then $R G$ is a right order in a right artinian ring.

Proof. Let $\gamma$ be an ordinal and let $\left\{G_{\alpha} \mid \alpha<\gamma\right\}$ be the ascending normal series of $G$ with $G_{0}=(1)$ and $G=G_{\gamma}$ as assumed. By transfinite induction we show that $R G_{\alpha}$ is a right order in a right artinian ring for every $\alpha \leqq \gamma$. 
Let $\lambda \leqq \gamma$ be an ordinal such that $R G_{\alpha}$ is a right order in a right artinian ring for all $\alpha<\lambda$. From Lemma 2.2 we obtain the result in case $\lambda$ is not a limit ordinal. Thus we may assume that $\lambda$ is a limit ordinal. Since the ascending normal series in $G$ has only a finite number of finite factors, there is an ordinal $\beta<\lambda$ such that all factors between $G_{\beta}$ and $G_{\lambda}$ are infinite cyclic. Therefore, applying Lemma 2.4, it follows that $R G_{\lambda}$ is a right order in $Q_{\lambda}=\lim _{\alpha<\lambda}\left(Q_{\alpha}\right)$. In particular $J\left(Q_{\lambda}\right)=J\left(Q_{\beta}\right) Q_{\lambda}$ is a finitely generated nilpotent right ideal of $Q_{\lambda}$ and $Q_{\lambda} / J_{\lambda}$ is a semisimple artinian ring. Hence $Q_{\lambda}$ is right artinian.

THEOREM 2.6. Let $R$ be a ring which is an order in a $Q F$ ring and let $G$ be a group which has a (transfinite) ascending normal series with each factor either finite or cyclic, but only a finite number of finite factors. Then $R G$ is an order in a QF ring.

Proof. As in the proof of Theorem 2.5 we proceed by transfinite induction and use the same meanings of $\alpha, \beta, \gamma, \lambda$. By Theorem $2.5 R G_{\alpha}$ is an order in an artinian ring $Q_{\alpha}$ for each $\alpha \leqq \gamma$. The case that $\lambda$ is not a limit ordinal results from Lemma 2.2. Now let $\lambda$ be a limit ordinal. Using the characterization of $Q F$ rings given by Hajarnavis (1971; page 336) it is sufficient to show that

(i) the left annihilator and the right annihilator of $J\left(Q_{\lambda}\right)$ in $Q_{\lambda}$ coincide.

(ii) $Q_{\lambda}$ is a direct sum of uniform right (and left) ideals.

The proof of (i) is routine applying Lemma 2.4 (c). Now we consider the decomposition $1=e_{1}+\cdots+e_{n}$ with primitive orthogonal idempotents $e_{1}, \cdots, e_{n}$ of $Q_{\beta}$. By the induction hypothesis $Q_{\beta}$ is a $Q F$ ring, whence $e_{i} Q_{\beta}$ is a uniform right ideal of $Q_{\beta}$ for $i=1, \cdots, n$. Therefore from Lemma 2.4 (b) it follows that $Q_{\lambda}$ is the direct sum of the uniform right ideals $e_{i} Q_{\lambda}$ of $Q_{\lambda}$. This finishes the proof of the theorem.

\section{References}

C. R. Hajarnavis (1971), 'Orders in $Q F$ and $Q F 2$ rings', J. Algebra 19, 329-343.

A. Horn (1973), 'Gruppenringe fastpolyzyklischer Gruppen und Ordnungen in Quasi-FrobeniusRingen', Mitt. Math. Sem. Giessen 100.

I. Hughes (1973), 'Artinian quotient rings of group rings', J. Austral. Math. Soc. 16, 379-384.

A. Kurosh (1956), The Theory of Groups, Vol. 2 (Chelsea, New York, 1956).

R. C. Shock (1972), 'Polynomial rings over finite dimensional rings', Pacific J. Math. 42, $251-257$.

L. W. Small (1966), 'Orders in Artinian rings', J. Algebra 4, 13-41.

Mathematisches Institut

Justus-Liebig-Universität

63 Giessen

Arndtstr. 2

West Germany 